\title{
Ornstein-Uhlenbeck-Lévy Electricity Portfolios with Wind Energy Contracting
}

\section{A Theoretical Approach}

\author{
Genaro Longoria ${ }^{1}$ (i) - Alan Davy ${ }^{1} \cdot$ Lei Shi $^{2}$
}

Received: 12 July 2018 / Accepted: 17 October 2018 / Published online: 10 November 2018

(C) Springer Nature Singapore Pte Ltd. 2018

\begin{abstract}
To leverage the potential of integrating renewable sources into electricity portfolios the risk and cost trade-off of intermittency needs to be assessed. From the perspective of a Load Serving Entity (LSE), this work present the theoretical implications of energy allocation from two type of markets: bilateral long-term contracts and real-time trading. The purchasing of energy on both markets and from two different sources: wind energy and conventional generation is formulated with a stochastic procurement model (SPM). The unexpected jumps of spot market prices are modeled with a mean-reverting Lévy process. The wind energy availability is modeled with multiplicative Brownian motion transformed to a Rayleigh probability density function. The risk assessment is defined by the efficient frontier and a user defined risk level. The SPM is tested numerically. The contracted share of wind power is found to range between $8 \%$ and $16 \%$. Moreover, the analysis shows the convergence of SPM to an optimal portfolio irrespectively of the wind farm autocorrelation decay rate.
\end{abstract}

Keywords Contract management $\cdot$ Electricity portfolio $\cdot$ Renewable energy sources $\cdot$ Risk analysis $\cdot$ Strategic planning

\section{Introduction}

Rising carbon taxes, climate awareness and technology breakthroughs, among others, had influenced the surge of intermittent resources integration into the electricity supply [1]. However, before renewables penetration had significant importance the open market imposed already challenging problems. A case study that serves as example is the California's electricity price turmoil of 2000 [2]. Despite the fact of its positive impact, green energy, has increased the complexity of procurement planning of Load Serving Entities (LSEs). Modern energy portfolios encompass conventional and renewable long-term energy contracts to reduce costs and hedge against price volatility. Just-in-time supply and demand unbalances can be corrected through procurement in the spot market (SM).

Genaro Longoria

glongoria@tssg.org

1 Telecommunications Software and Systems Group, Waterford Institute of Technology, Carriganore, Co., Waterford, Ireland

2 Carlow Institute of Technology, Carlow, Co., Carlow, Ireland
In the pre-renewables era load forecasting was often the foremost concern to maintain the grid operating at acceptable frequency and voltage ranges. Works like [3] have contributed to pave the way to reliable load forecast methods. Nonetheless, developed countries are stretching the targets of renewable supply. The non-deterministic nature of future events, such as fossil-fuel prices and weather conditions, calls for strategic electricity portfolios to hedge volatility. Therefore, an important concern for a LSE is to determine the proportions of conventional and/or renewable to total demand. Possible alternatives to integrate variable renewable sources, within and external to the energy supply-chain, have been studied by Lund et al. [4].

This paper presents, from the perspective of an LSE, the energy allocation strategies from two type of markets, bilateral long-term contracts (LT) and real-time trading. A Stochastic Procurement Model (SPM) based on an Ornstein-Uhlenbeck-Lévy process is formulated to describe the uncertainty prevailing in future energy prices and in the availability of wind. A mean-reverting Lévy process is used to model the sudden changes of electricity prices. Wind speed is modeled with a stochastic differential equation (SDE) with Brownian noise. The latter is adapted to comply with given probability density function (pdf). The 
proposed SPM is discretized and solved numerically. Then, the different procurement strategies are assessed within the framework of Portfolio theory [5]. The risk analysis concurrently evaluates the energy mix. To complement CVaR we propose a metric such that the portfolio manager can establish the risk threshold. The assessment is concluded with the portfolios' efficient frontier and the corresponding probability for the aforementioned threshold. Our contribution is a readily implementable methodology that can serve an LSE to define the optimal energy mix and to quantify the trade-off between risk and cost.

Previous work has considered the uncertainty, in wind and spot price, through a finite number of scenarios. Several studies have shown that wind speed can be approximated by a Weibull distribution or its particular case, the Rayleigh distribution (RD) [6-8]. On the other hand, as already highlighted in the work of [9], a sound energy price differential equation should incorporate not solely a Brownian-like motion but possible spikes along the trajectory. A meanreverting Lévy process models sudden price jumps in the spot market.

The remainder of the paper is organized as follows. Section "Related Work" presents a brief literature review, comment on the related work and state our contributions. Followed by the problem statement in "Problem Statement". In "Stochastic Procurement Model" and "Simulation and Discretization Method" the SPM and simulation method is presented, respectively. The risk assessment is detailed in "Portfolio Risk Analysis". The testbed and numerical results are analyzed in "Experimental Validation". Finally, "Conclusion" conclude the work done.

\section{Related Work}

A thorough overview of modern electricity market-design in the United States and across the European Union can be found in the surveys of Bushnell et al. [10] and Scharff and Amelin [11], respectively. The open market has a myriad of challenges to be address. For example, distributed generation (DG) poses the problem that no single entity supplies the aggregated demand. Thus, system operators need to reserve ancillary services in order to maintain the entire grid within safety operational levels[12]. On the other hand, DG has created pricing zones. Pricing schemes for up and down regulation of ancillary services, unit commitment and secure operation of the grid raises concerns about opportunity costs for both suppliers and consumers. The paper [13] presents a solution to locational marginal prices. The authors use Benders decomposition and Lagrangian optimization, they prove better performance with respect to other methods and fairness towards suppliers of spinning reserves. The concept of fairness is a preponderant constraint such that the market can remain competitive and stable. Our work serves firstly LSEs and indirectly the system operator by reducing the gap between real-time supply and demand thus lessening spinning reserves dependency.

The problem of optimizing the energy mix, among bilateral contracts, self-production and the SM, for a large consumer is studied by [14]. They developed a linear programming model to minimize the consumer's electricity bill. They considered conventional generation whereas we also address the problem of integrating an intermittent source or energy. More recently, [15] discussed the application of financial risk methods in electricity procurement. In the paper, they converted the cost and risk problem into a single optimization model.

The literature of bilateral or forward contracts has traditionally considered the fossil-fuel type of generation. In [16], Kneževic et al., under the assumption of perfect inelastic demand, simulated three cases of energy pricing and contracting. They concluded that the scenario with bilateral contracts is of mutual interest for generation and demand. Whereas [17], proposed an on-the-fly demand allocation adjusting scheme. A penalty was used to compensate the generation company for positive difference between energy contracted and consumed. In this context, the earlier work [18] developed a multi-stage algorithm to modify electricity delivery from a bilateral contract. A risk measure is used in the work of [19]. The paper asses forward contracts against spot market procurement on the basis of Insufficiency of Load Supply. Because of the volatility of the energy market and load demand, they adopted a Monte Carlo (MC) method to estimate the buyer's risk exposure. Thereafter they determined the next contracts negotiating conditions. In earlier studies, [20] presented a game theoretic approach to the portfolio creation for multi energy wholesalers and buyers. Whereas, [21], with a similar theoretic background, determined the equilibrium contracting electricity price.

The recent work [22] presented a method for coalitions of renewable producers. They used cooperative game theory and applied the Nucleolus allocation method. A main contribution of the method is to make computational tractable the problem of maximizing the worst-case quota of the aggregated generation. Nevertheless, the authors considered a fixed set of scenarios. In a similar setting, although different value function, [23] have previously analyzed the allocation strategies of aggregated bidding. The paper shows the existence of a payoff such that coalition stability is assured and hence an increase of expected profit.

These literature has approached the dynamics underlying the uncertainty mostly through fixed scenarios. The work presented here can serve as bridge between randomness modelling and electricity contracting.

The SPM is reliable in that it quantifies the long-term cost and risk of procuring energy from intermittent resources. 
The SPM can scale up to incorporate other type or energy sources. It can be easily tailored to the particularities faced by the LSE. The SPM formulation is also flexible to explore different portfolios. Our contribution to electricity contract management moves forward the state-of-the-art of the commonly adopted finite scenario analysis by proposing the SPM.

\section{Problem Statement}

In the short term the renewable/fossil-fuel ratio is expected to expand significantly [24]. To address the uncertainty of renewable energy resources the SPM quantifies the risk upon profit. The paper present a model that can be easily tailored to any particular market context. An LSE can procure electricity in the power pool and from forward bilateral contracts with conventional and renewable energy wholesalers. The LSE requires reliable estimates of potential renewable generation and market trading prices prior to determine its demand allocation strategy.

Electricity markets are assumed frictionless, this means that there are no transaction cost or differential taxes. Prices are considered to be the result of a large number of high frequency small perturbations happening through time. At a given moment $t$ of the trading day, the market price is the result of marketeers' buy and sell bids, which the system operator uses to calculate the energy marginal price. PJM defines the locational marginal price as the "cost to serve the next MW of load at a specific location, using the lowest production cost of all available generation" [25].

The model optimizes the total cost of procuring electricity (CEP) for a given portfolio. Bilateral contract in the form of Options are composed of two types of costs, i.e., reservation and execution cost. The reservation cost, assures the LSE an amount of power during the trading day at an agreed timeframe. Although the LSE can decide to partially or not make use of it subject to paying the execution price [26]. Moreover, the CEP takes into consideration the penalty imposed by the Independent System Operator (ISO). Mismatches incur in an under-delivery fee of committed supply. Lastly, prior to dispatch time the LSE must submits to the CG and ISO the next period's executed energy and real-time procurement.

\section{Stochastic Procurement Model}

An Ornstein-Uhlenbeck-Lévy process is formulated to approach the randomness of intermittency and market volatility. Geometric Brownian motion is limited in modelling leptokurtic distributions and price clustering effects [27].
A Lévy process is used to obtain realistic price dynamics. Along with the Brownian motion, a compound Poisson process is coupled to account for "rare" events. These unexpected events are beyond the day-to-day marketeers interactions, instead they are due to sudden regional or global changes. Hence, the SPM couples a mean-reverting jump and drift-diffusion SDEs to quantify daily market "collisions" and exogenous events of the electricity price.

Literature reviews and site studies have shown the applicability of polynomial forms as estimates for the dependency of power upon wind speed [6, 28]. In our work, the energy procured from a WPP is obtained by numerically solving a transformed SDE.

\section{Lévy Driven Price Dynamics}

The spot price is represented by $p_{s m}(t)$. Let $(\Omega, \mathscr{F}, \mathbf{P})$ be a complete probability space on which the stochastic processes are defined. Where $\left\{\mathscr{F}_{t}\right\}_{t \in[0, T]}$ is the information available up to time $t$. Let $\mathbb{E}$ denote expectation under probability measure $\mathbf{P}$. The convention that the underlying process is right continuous is used to account for unexpected price increments. This can be expressed as $p_{s m}(t)=$ $\lim _{u \downarrow t} p_{s m}(u)$.

A Poisson counting measure $N(t)$ has memory-less of the renewal process, reflected on the i.i.d. interarrival times. The SPM exploit this feature to account for unexpected electricity price spikes. The probability of an event occurring during a finite time interval $\Delta$ can be described as

$$
\begin{aligned}
& \operatorname{Prob}\{\neg \text { event in }(t, t+\Delta)\}=1-\lambda \Delta+\mathscr{O}(\Delta), \\
& \operatorname{Prob}\{\text { one event in }(t, t+\Delta)\}=\lambda \Delta+\mathscr{O}(\Delta), \\
& \operatorname{Prob}\{\text { more than one event in }(t, t+\Delta)\}=\mathscr{O}(\Delta),
\end{aligned}
$$

where $\lambda$ is the intensity or jump rate. The probability of $n$ jumps taking place in the interval $\Delta=t-s$, is given by

$P(N(t)-N(s)=n)=\frac{e^{-\lambda \Delta}(\lambda \Delta)^{n}}{n !}$,

where $0 \leq s<t . N(t)$ represents the total number of events that have occurred up to time $t$ and is constant between any two consecutive jumps. From Eq. 1 it can be seen that increments are exponentially distributed, same sized and with negligible probability of more than one jump in the same time interval.

The SM price of electricity just before a jump is denoted by $p_{s m}(t-)$. Then $p_{s m}(t)$ is given as the limit from the left, i.e., $p_{s m}(t-)=\lim _{u \uparrow t} p_{s m}(u)$.

Spot price spikes are characterized by its martingale evolution. For a jump process to exhibit those properties a compensator for $N(t)$ must be included. The martingale version (or in this case a right continuous martingale) is a compensated Poisson process given by $J(t)=N(t)-\lambda t$, 
such that $\mathbb{E}\left[N(t)-\lambda t \mid \mathscr{F}_{t}\right]=0$. The latter implies that jumps exhibit no trend, i.e., they are totally unpredictable, irrespective of the time interval size.

The power-market electricity price jump-drift-diffusion model, with initial condition $p_{s m}(0)=p_{o}$, drift and diffusion $\mu_{s m}$ and $\sigma_{s m}$ respectively and $t \in[0, T]$ is given by

$\frac{d p_{s m}(t)}{p_{s m}(t-)}=\gamma\left[\mu_{s m}-\lambda \log p_{s m}(t)\right] d t+\sigma_{s m} d B(t)+d J(t)$.

Equation 2 is a mean-reverting with additive noise SDE. The first two terms in Eq. 2 represent the OrnsteinUhlenbeck process where $\gamma$ is the rate towards the longrange mean $\mu_{s m}$ and the Brownian motion $d B(t)$ accounts for the energy traders interactions. Lastly, $d J(t)$ is the Lévy process to model sudden short-lived price escalations.

Furthermore, for a realistic price model the SPM has a non-constant jump size. A Poisson process with variable increments is called a Compound Poisson process. Following the derivation presented by [29], let us define the size of the jump as $Y_{j}-1$ where $Y_{1}, Y_{2}, \ldots$ are i.i.d. random variables independent of $N(t)$ and $B(t)$. The change $d p_{s m}$ of $p_{s m}$ depends on its value before the jump. The increment at the time of a jump is $p_{s m}(t)-p_{s m}(t-)$. This change is different from zero only if $J$ jumps at $t=t_{j}$, then

$$
\begin{aligned}
p_{s m}\left(t_{j}\right)-p_{s m}\left(t_{j}-\right) & =p_{s m}\left(t_{j}-\right)\left[J\left(t_{j}\right)-J\left(t_{j}-\right)\right] \\
& =p_{s m}\left(t_{j}-\right)\left(Y_{j}-1\right) .
\end{aligned}
$$

From Eq. 3 it is straightforward to show that $p_{s m}\left(t_{j}\right)=$ $p_{s m}\left(t_{j}-\right) Y_{j}$. To obtain a solution to the price SDE, we can try an ansatz of the form $X(t)=X(0) e^{\mu t}$ and use Itô Integral. To account for the non-zero first moment of $p_{s m}$, Itô calculus' random term $d B^{2}(t)$ is proportional, in a mean square sense, to $d t$ [30]. Equation 2, is justified as a symbolic representation of

$$
\begin{aligned}
\int_{t}^{t+\delta} \frac{d p_{s m}(s)}{p_{s m}(s-)}= & \gamma \int_{t}^{t+\delta}\left[\mu_{s m}-\lambda \log p_{s m}(s)\right] d s \\
& +\int_{t}^{t+\delta} \sigma_{s m} d B(s)+\int_{t}^{t+\delta} d J(s),
\end{aligned}
$$

because Brownian's motion unbounded variation is nowhere differentiable. The first term on the RHS is understood in the Riemann sense and $\delta$ is an infinitesimal time. To solve for $p_{s m}(t)$, Itô-Doeblin formula for one jump process is used [31]:

$$
\begin{aligned}
f(X(t))= & f(X(0))+\int_{0}^{t} f^{\prime}(X(s)) d X^{c}(s) \\
& +\frac{1}{2} \int_{0}^{t} f^{\prime \prime}(X(s)) d X^{c}(s) d X^{c}(s) \\
& +\sum_{0<s \leq t}[f(X(s))-f(X(s-))],
\end{aligned}
$$

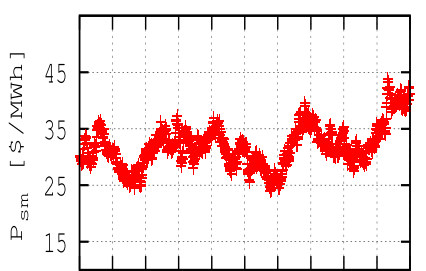

Time
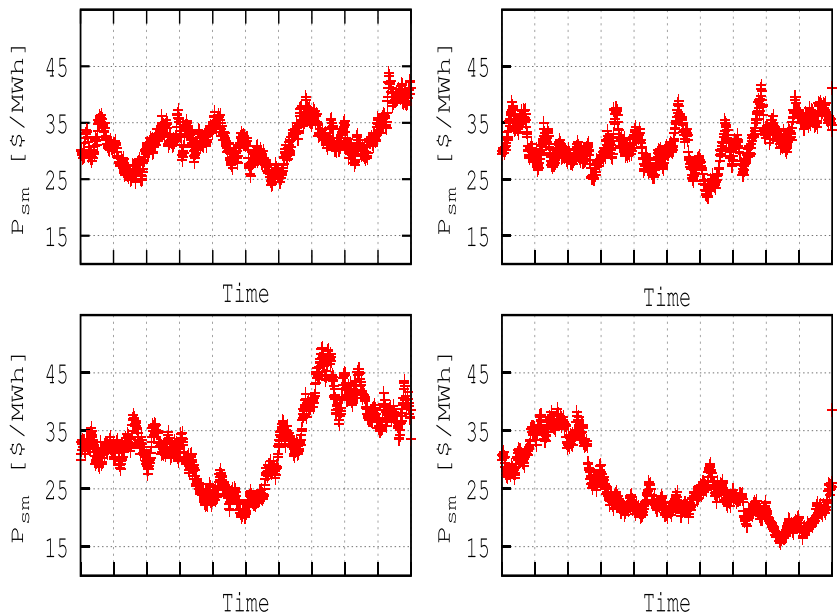

Time

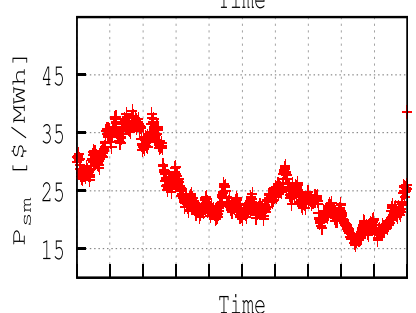

Fig. 1 Typical realization paths of the SDE (2) of $p_{s m}(t)$. The plots present four different electricity price paths per MWh dispatched according to equation (5). The paths reflect the Lévy and OrnsteinUhlenbeck processes characteristic of electricity prices

$X^{c}(t)$ is the continuous part of a Lévy process $X(t)$. Using $\Phi(t)=\log P_{s m}(t)$, the solution to (2) can be obtained from

$$
\begin{aligned}
\Phi(t)= & e^{-\gamma t} \Phi(0)+\gamma \mu_{s m} \int_{0}^{t} e^{-\gamma(t-s)} d s \\
& +\sigma_{s m} \int_{0}^{t} e^{-\gamma(t-s)} d B(s)+\sum_{j=N(t)+1}^{N(t+1)} \log \left(Y_{j}\right) .
\end{aligned}
$$

To derive Eq. 5 we used the ansatz, Itô Integral and the fact that $\log \left(p_{s m}\left(t_{j}\right)\right)=\log \left(p_{s m}\left(t_{j}-\right)\right)+\log \left(Y_{j}\right)$ such that $f(X(t))=\log \left(p_{s m}(t)\right)$ in Eq. 4.

Figure 1 shows typical realizations of spot market hourly price, $p_{s m}(t)=\exp [r(t)]$, of electricity dispatched. The effect of the mean-reverting term can be seen on the comeback-to-trend after a price spike.

\section{Transformed Stochastic Wind Dynamics}

The electricity generation of a wind farm depends on, e.g., blade design, gearbox, hub height. Nevertheless, the largest impact variable is wind speed [32]. In wind turbine design and technical literature, a widely used relation between wind speed $v$ and power $w$ is the cubic power law [6]:

$w(v(t))=\frac{1}{2} \rho A C_{p} \eta v^{3}(t)$.

where $A$ is the swept area of the rotor in $m^{2}, \eta$ is the efficiency of the wind turbine, $\rho=1.225 \mathrm{~kg} / \mathrm{m}^{3}$ is the air density at mean sea level and $C_{p}$ is the rotor efficiency [33].

The map from wind speed to power is done using Eq. 7. This model can be easily parametrized to an specific WPP.

$d v(t)=\mu_{w}(v(t), t) d t+\sigma_{w}(v(t), t) d B(t)$ 
The functions $\mu_{w}(\cdot)$ and $\sigma_{w}(\cdot)$ are expressed in terms of a given pdf to resemble the distribution and autocorrelation of the wind speed statistics. The method presented in [34] relates the SDE coefficients and a probability density, $p(\cdot)$, through the Fokker-Planck differential equation,

$$
\begin{aligned}
\frac{\partial p(v(t), t)}{\partial t}= & -\frac{\partial}{\partial v(t)}\left[\mu_{w}(v(t), t) p(v(t), t)\right] \\
& +\frac{1}{2} \frac{\partial^{2}}{\partial v^{2}(t)}\left[\sigma_{w}^{2}(v(t), t) p(v(t), t)\right] .
\end{aligned}
$$

The randomness associated with the stochastic quantity is in a steady state phase hence we consider the case of stationary processes. In other words, random events are time invariant and second order measures depend only on time differences [35]. Thus, Eq. 8 can be written as

$$
\frac{\partial}{\partial v(t)}\left[\mu_{w}(v(t)) p(v(t))\right]=\frac{1}{2} \frac{\partial^{2}}{\partial v^{2}(t)}\left[\sigma_{w}^{2}(v(t)) p(v(t))\right] .
$$

To closely resemble any site specific wind speed behavior, the autocorrelation resulting from the SDE should follow that of a wind distribution. Zárate-Miñano and Milano's method is motivated by the Regression theorem applied to a Markovian process [30]. Firstly, the drift term is determined. This is done by using Itô's lemma and the stationary properties of the second order moments. Then, the autocovariance differential equation of the underlying stochastic process is solved. Lastly, the drift is adapted to the desired exponential decay. After integration of Eq. 9, the method solves for the diffusion term.

It has been compared how closely the Weibull and its particular case the RD fits speed data. For instance, [36] found a more accurate data fit from the Weibull pdf over RD. They estimated, for a particular month, the RMS deviation as 0.0031 and 0.0073 , respectively. On the other hand, [6], [7] and [33] argued differently. The work presented uses the RD since wind speed is location dependent and to keep the exposition easy to follow.

For the one parameter, $\gamma$, RD with pdf given by $f(v)=$ $\frac{2 v}{\gamma^{2}} \exp \left[-(v / \gamma)^{2}\right]$ the drift and diffusion are expressed as

$\mu_{w}(v(t))=-\alpha\left(v(t)-\gamma \sqrt{\frac{\pi}{2}}\right)$

and, where $\alpha$ is the autocorrelation decay rate,

$$
\begin{aligned}
\sigma_{w}(v(t))= & \sqrt{\frac{\alpha \gamma^{2}}{v(t)}} \\
& \times \sqrt{\left[2 v(t)+\gamma \sqrt{2 \pi}\left(e^{\frac{v^{2}(t)}{2 \gamma^{2}}} \operatorname{erfc}\left(\frac{v(t)}{\gamma \sqrt{2}}\right)-1\right)\right] .} .
\end{aligned}
$$

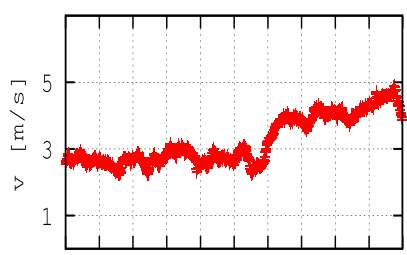

Time
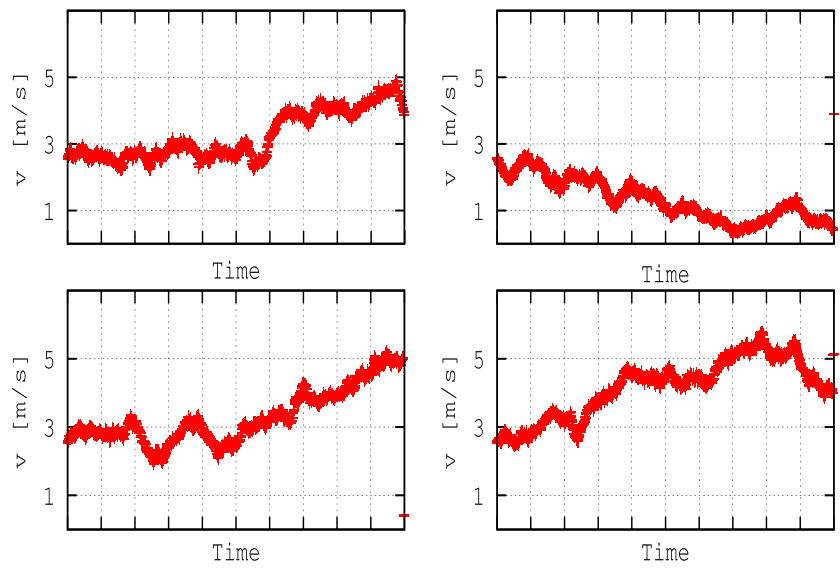

Time

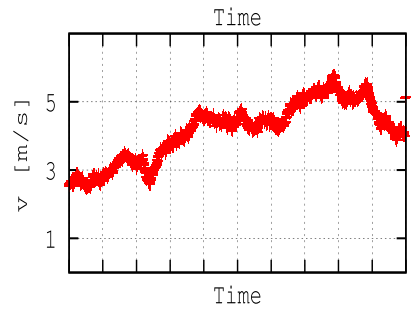

Fig. 2 Typical realization paths of $v(t)$. The wind speed paths are the solution to a transformed SDE such that the autocorrelation is that of a Rayleigh distribution

An algorithm to solve (7) can be derived from integrating both sides of the SDE, i.e.,

$v(t)=v(0)+\int_{0}^{t} \mu_{w}(v(s)) d s+\int_{0}^{t} \sigma_{w}(v(s)) d B(s)$

Section 2 presents a 4th order Runge-Kutta time discretization scheme to solve the SDE (12). Figure 2 shows four different wind realizations according to Eq. 7 with drift and diffusion given by Eqs. 10 and 11 respectively.

\section{Energy Procurement Cost}

In this section we present a methodology to determine the energy mix that minimizes procurement cost. The LSE is considered to be subject to penalty fees for under fulfillment of the committed demand.

Wind power is a competitive source for electricity generation. The variability and the inability to dispatch at command can counteract the benefits of its renewable nature. A major benefit of LT is price stability in an arbitrage-free framework [27]. In this context, a forward contract with bilateral options entitles the buyer the right to partially exercise the contracted electricity, albeit a fixed reservation cost.

Equation 13 is the total cost of procuring electricity, CEP. It is composed of

$\xi$ the minimum, at delivery time $t$, of $d_{w}$ and $w(v)$, i.e., electricity contracted and available, respectively, bought at $p_{w}$, the WPP selling price;

execution and reservation costs, $g$ and $s$ respectively and the share $d_{c}$ of electricity allocated to a CG;

$x$, the energy bought from the spot market at price $p_{s m}$; lastly, depending on the wind power generation $\zeta$, a penalty $\mathscr{P}$ for under fulfillment of the LSE committed total demand $D$.

$\mathrm{CEP}(t)=\xi p_{w}+d_{c} g+s\left(d_{c}\right)+x(t) p_{s m}(t)+\mathscr{P} \zeta p_{s m}(t)$ 
$D=d_{w}+d_{c}+x(t)$

$\xi=\min \left[d_{w}, w(v(t))\right]$

$\zeta= \begin{cases}0 & \text { if } w(v(t-1))<d_{w} \\ \max \left[D-w(v(t))-d_{c}, 0\right] & \text { if } w(v(t-1))>d_{w}\end{cases}$

$x(t)= \begin{cases}0 & \text { if } p_{s m}(t-1)>s\left(d_{c}\right)+g \\ D-d_{w} & \text { if } p_{s m}(t-1)<s\left(d_{c}\right)+g \\ d_{w} & \text { if } w(v(t-1))<d_{w} .\end{cases}$

The LSE electricity adjustment process is bound to the period before the next delivery time. This means that the next period executed conventional energy and real-time procurement have to be notified prior to dispatch time. This is motivated by the martingale assumptions about wind speed and market price dynamics. The decision-making algorithm of our model (13) is shown in Fig. 3. The flowchart diagram is the LSE electricity allocation algorithm. At trading session the algorithm adapts the LSE short-term procurement

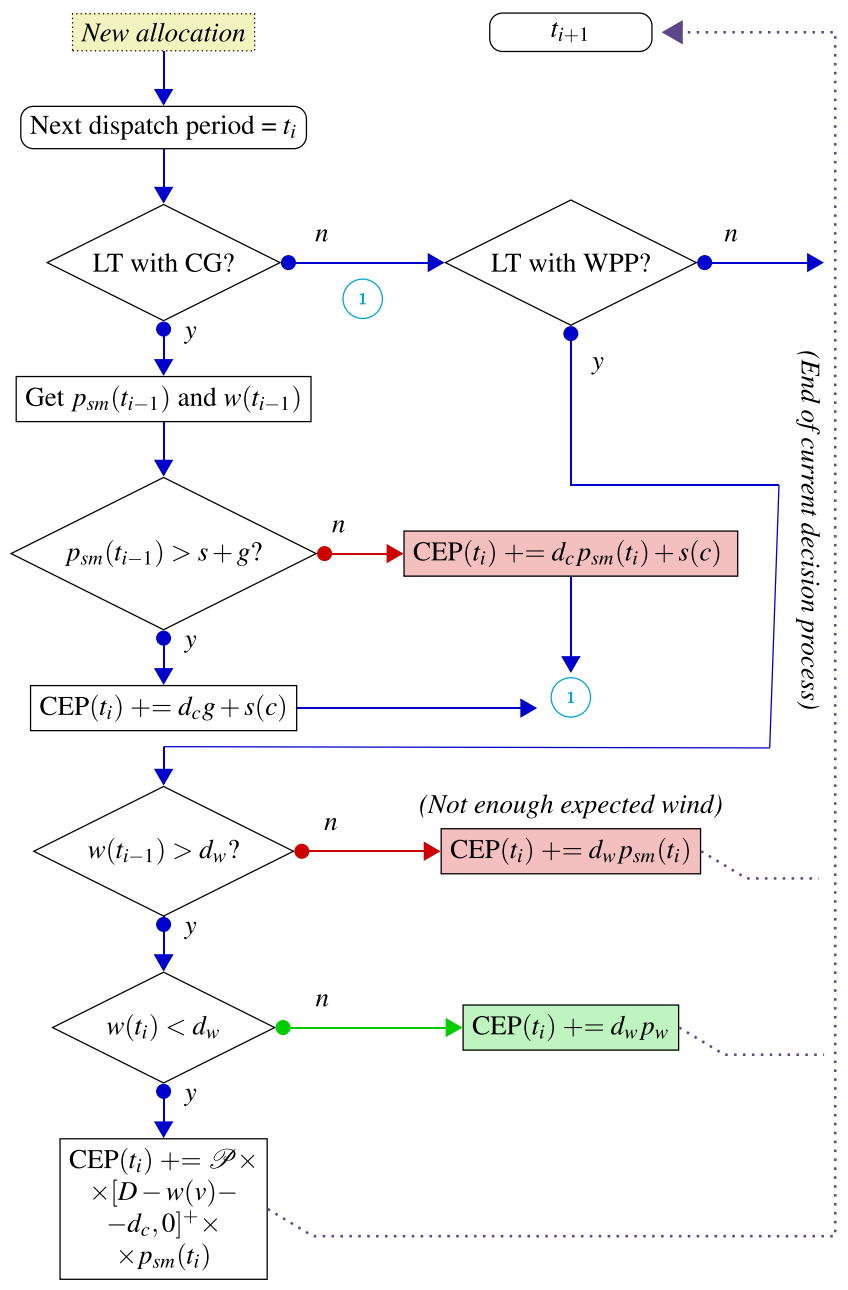

Fig. 3 Flowchart diagram of a LSE electricity procurement decision support algorithm. The energy portfolio is composed of real-time trading in the exchange market and two bilateral contracts with a conventional and a renewable source, respectively strategy, such that, in the long-term the energy contracts and intraday adjustments minimize penalty fees and procurement costs.

\section{Simulation and Discretization Method}

The dynamical portfolio of the LSE assumes an amount allocated in a riskless asset and the rest of its demand in a risky electricity generator. This work implements a MC approach to estimate expected values for the LSE procurement costs. The length of the period $[0, T]$ is segmented into $m$ equal parts. The time step is $h=T / m$ such that $0=t_{o}<h<\cdots<t_{m}=T$ and $\Delta T=t_{i+1}-t_{i}=h$.

To determine the price dynamics a recursive scheme solves (5). The discretization routine is

$$
\begin{aligned}
\Phi\left(t_{i+1}\right)= & e^{-\gamma t} \Phi\left(t_{i}\right)+\mu_{s m}\left(1-e^{-\gamma t}\right) \\
& +\sigma_{s m} \sqrt{\frac{1-e^{-2 \gamma \Delta t}}{2 \gamma}} Z+M,
\end{aligned}
$$

where $Z \sim \mathscr{N}(0,1)$ and $M$ is the summation of the logarithm of $N$ Poisson distributed jumps [29]. To solve the SDE for the speed of wind, Eq. 12 is numerically integrated with the stochastic Runge-Kutta scheme (14). The value of the coefficients $\varphi_{p}, c_{p}$ and $a_{p q}$ and the covariance among the $z_{q}$ values are those derived in Kasdin's fourth order $(n=4)$ explicit Runge-Kutta method [37].

$$
\begin{aligned}
v\left(t_{i+1}\right)= & v\left(t_{i}\right)+\varphi_{1} k_{1}+\varphi_{2} k_{2}+\cdots+\varphi_{n} k_{n} \\
k_{1}= & h \mu_{w}\left(t_{i}+c_{1} h\right) v\left(t_{i}\right)+h \sigma_{w}\left(t_{i}+c_{1} h\right) z_{1} \\
k_{q}= & h \mu_{w}\left(t_{i}+c_{q} h\right)\left(v\left(t_{i}\right)+\sum_{p=1}^{q-1} a_{p q} k_{p}\right) \\
& +h \sigma_{w}\left(t_{i}+c_{q} h\right) z_{q} .
\end{aligned}
$$

A principal virtue of the method, compared to an EulerTaylor approximation, is to avoid computing the derivatives of the drift and diffusion coefficients. This is achieved without sacrificing order of convergence. On the other hand, the drawback is the required multi-step calculations. Nevertheless, given Eqs. 10 and 11 a derivative free method is preferred.

\section{Portfolio Risk Analysis}

The penetration of intermittent energy resources and distributed technologies is increasing the complexity of the risk diversification task. For a reliable estimation of future profits the extent of potential non-desirable extreme events has to be considered. This work complements the portfolios with two risk measures. Firstly, Conditional Value-at-Risk (CVaR) analyzes the relation between risk and costs. Based 
upon the CVaR measure, the efficient frontier is calculated for various possible diversification schemes. Lastly, the Excess Cost metric is formulated. The latter risk measure contrasts the cost of electricity procurement for different portfolios with the probability of exceeding a given cost threshold.

\section{Risk Metric: CVaR}

The uncertainty prevailing in commodity markets might negatively transfer the price volatility to the LSE procurement cost if the portfolio risk is not considered. It has been demonstrated that CVaR quantifies the risk of the electricity portfolios.

Among other desired properties, $\mathrm{CVaR}$ is a coherent metric. Specifically it complies with the subadditivity property. This means that, for any portfolio composed of a linear combination of other portfolios, the risk is not overestimated [38].

Lastly, another advantageous characteristic is that the formerly popular, but not coherent, Value-at-Risk (VaR) measure is a byproduct of optimizing CVaR; as can be seen in Eqs. 15 and 16.

$\mathrm{VaR}=\min \{v: P(\mathrm{CEP}(t) \leq v) \geq \beta)\}$

$\mathrm{CVaR}=v+\frac{1}{1-\beta} \mathbb{E}\left[(\operatorname{CEP}(t)-v)^{+}\right]$,

where $v$ represents the value-at-risk, $\beta$ is a specified confidence level for the probability of $\operatorname{CEP}(t)$, typical values are $0.9,0.95$ and 0.99 . The $(x)^{+}$notation stands for the maximum among $x$ and 0 .

\section{Risk Metric: Excess Cost}

This work proposes a risk metric that is based on the cumulative probability exceeding a defined threshold. In this context, risk can be understood as the expected energy procurement cost in excess of a zero-risk portfolio. The histogram in Fig. 4 is an example of the idea behind our Excess

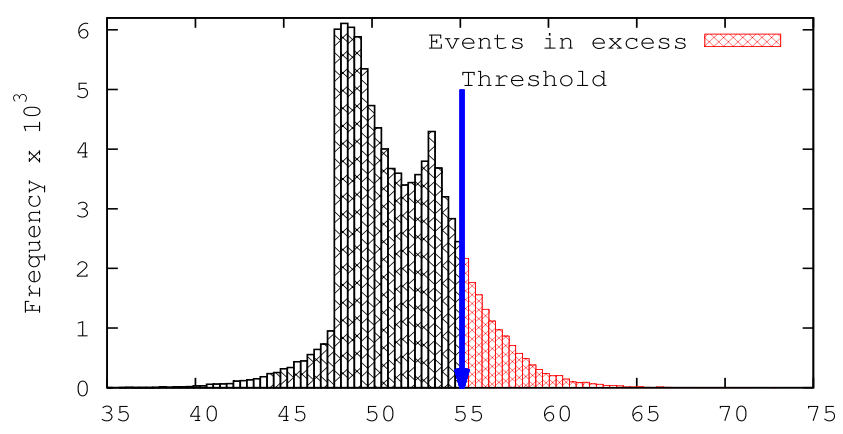

Fig. 4 A process dependent on a random variable can be evaluated by the probability that the random variable surpasses a threshold
Cost metric. Excess Cost is a quantitative evaluation of the probability of events occurring with a negative impact on the LSE profit.

An LSE riskless strategy, although expensive, would be to procure the total demand $D$ from a LT with a dispatchable source. This sets the benchmark such that the LT electricity price with a CG be the threshold. For every portfolio Excess Cost quantifies the probability that the cost surpasses this threshold.

\section{Experimental Validation}

The applicability of the SPM algorithm is shown by simulating for $m=1000$ and $\gamma=3.4$. The time step $h=0.001$, represents the time slots during a trading day for contract adjustments and hourly bids. The statistics are the outcome of 100000 paths. Table 1 , summarizes the wind turbine specifications of the WPP [6]. A highly liquid energy exchange [39] is used as reference of the electricity price. The LSE total demand is $D=2 \mathrm{MWh}$ and contract prices are $p_{w}=18 \$$ and $g=24 \$$ per MWh.

The SPM and risk analysis are validated with a Monte Carlo algorithm. The modelling and simulations are coded in $C$ programming language. The experimental setup was executed on Ubuntu-14.04-trusty-server-x86_64 with $16 \mathrm{~GB}$ RAM and 2.6 GHz QuadCore Intel Xeon E312xx (Sandy Bridge).

\section{Results and Analysis}

The threshold $\theta$ is defined as the total procurement cost under a pure CG portfolio. Figure 5 shows the expected cost, $\mathbb{E}[\mathrm{CEP}]$, for different electricity portfolios and distinct decay rates, $\alpha$. It can be seen the possible wind energy diversification strategies that will keep procurement cost below $\theta$. Values above the solid line mean that, in the long run, the uncertainty in the wind power availability, either from low or extremely high wind speeds, counteracts the price advantage of the renewable source. For $\alpha=0.7$, the minimum expected costs comprises $16 \%$ energy from the WPP.

Table 1 Wind turbine data used in the MC simulations

Wind Turbine Specs

\begin{tabular}{ll}
\hline Rated power & $1.3 \mathrm{MW}$ \\
Rated speed & $15 \mathrm{~m} / \mathrm{s}$ \\
Cut-in speed & $4 \mathrm{~m} / \mathrm{s}$ \\
Cut-out speed & $25 \mathrm{~m} / \mathrm{s}$ \\
Swept area & $3019.5 \mathrm{~m}^{2}$
\end{tabular}




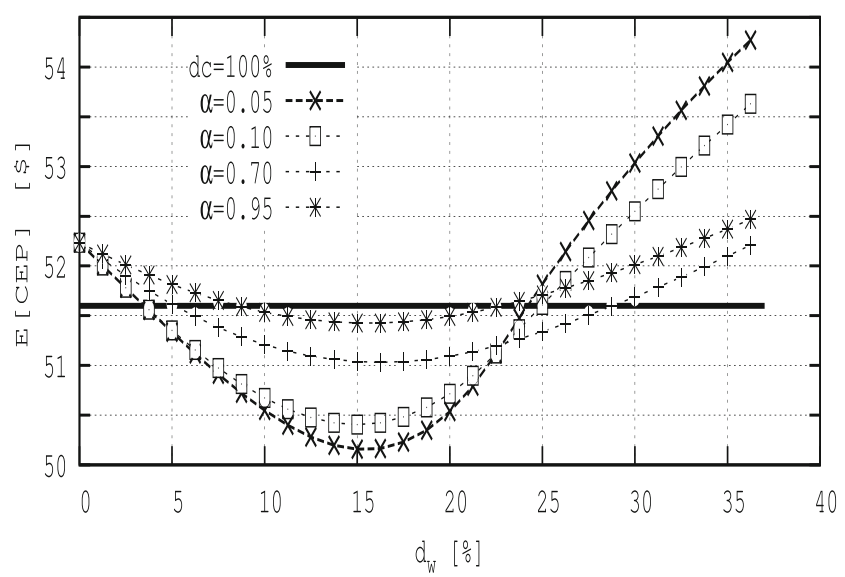

Fig. 5 Effect of $\alpha$ on the expected procurement cost for different $d_{w}$

The non-linear dependence of the minimum $\mathbb{E}[\operatorname{CEP}(t)]$ on the wind correlation decay rate is shown in Fig. 6 . The initial steep increase of the minimum expected cost reflects the strong dependence upon lower values of $\alpha$. Nevertheless, there exist a diversification scheme such that the WPP can be profitably incorporated; in our testbed it spans from $15 \%$ to $18 \%$. This means that for different $\alpha$ 's, the LSE can allocate its electricity demand, among the WPP a CG and the SM, such that the minimum expected procurement cost is less than $d_{c}=100 \%$.

Figure 7, is the procurement cost and risk map for the LSE. The graph shows that lower CVaR levels can be obtained by sacrificing (i.e., increasing) the portfolio's expected cost.

CVaR risk is the expected payable cost in the worst $10 \%$ and $5 \%$ cases $(\beta=.9, .95)$. Figure 7 shows that for a greater confidence level, higher cost are expected from within the profit adverse events. Thus, for a risk averse LSE, the minimum CVaR is achieved with a $8 \%$ share of wind electricity.

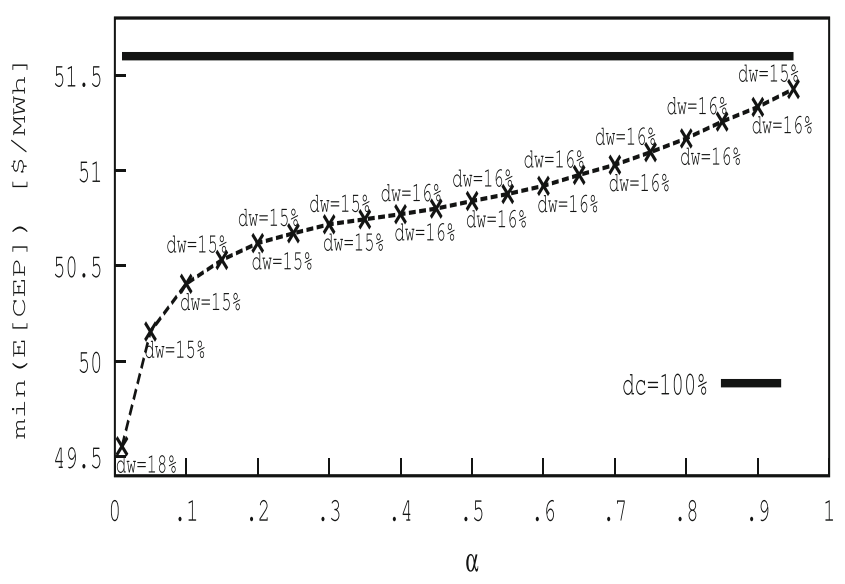

Fig. 6 Minimum $\mathbb{E}[\mathrm{CEP}]$ the $\%$ of $d_{w}$ for different $\alpha$

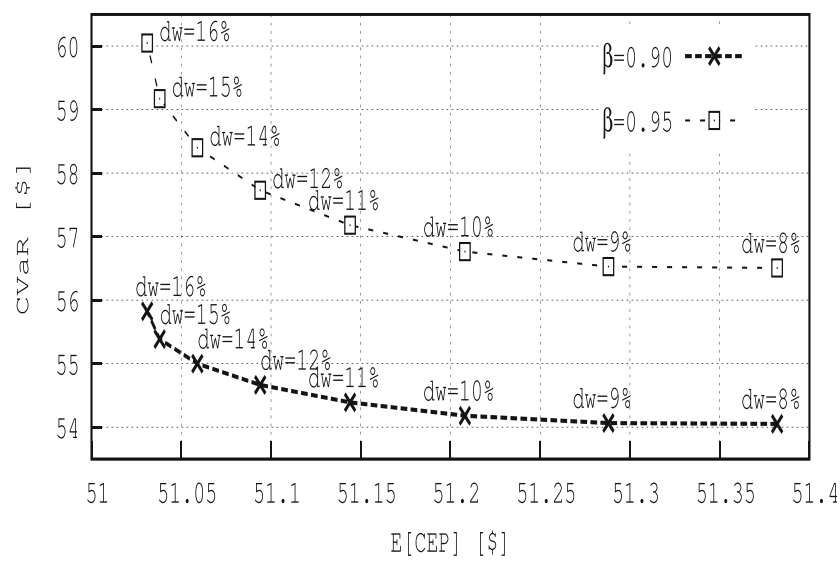

Fig. 7 Efficient frontier for different $d_{w} . \alpha=0.7$ and two $\beta$ levels

The Excess Cost assessment can be seen in Fig. 8 . Under this measure, the portfolio with minimum probability of events above $\theta$ is comprised of $11 \%$ electricity from the WPP. This corresponds to the inflection point where increasing the share of renewable energy no longer benefits profit creation.

\section{Discussion}

The factors determining the renewable share that minimizes risk are, besides the skewness and location of the peak, the kurtosis and spread of the procurement cost. This can be seen in Fig. 9d. The backbone of the SPM is the martingale adapting scheme. Figure 9 shows histograms for the three aforementioned renewable allocation schemes. In this respect, $\mathrm{CVaR}$ sheds light on the average tail events. On the other hand, Excess Cost provides an insight into the likelihood of a given portfolio incurring in high cost procurement.

Moreover, Excess Cost is understood as a trade-off among the relaxed and conservative hedged strategies. This

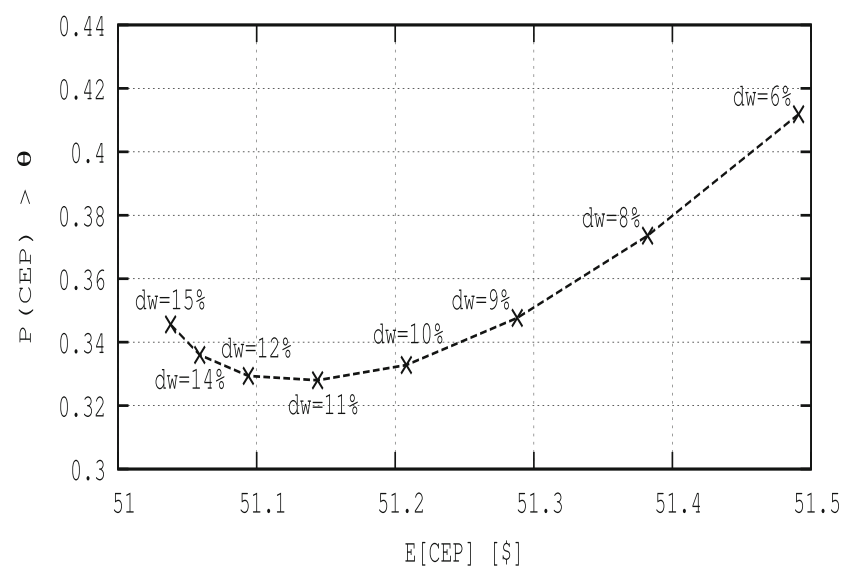

Fig. 8 Excess Cost metric for $\theta=51.6 \$$ and $\alpha=0.7$ for different $d_{w}$ 


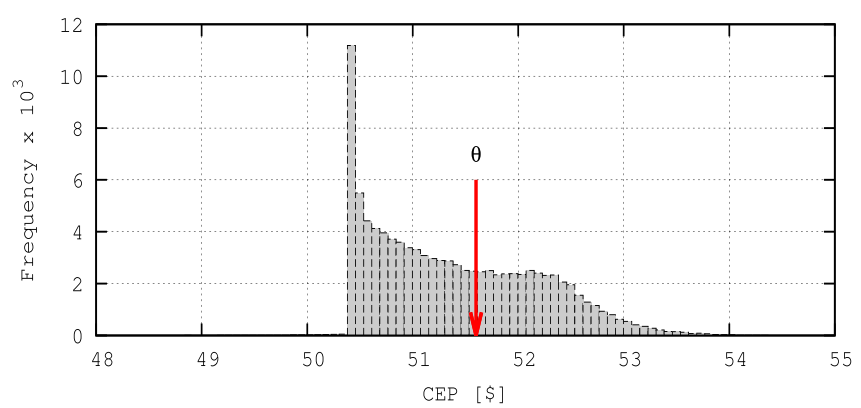

(a) $d_{w}=16 \%$

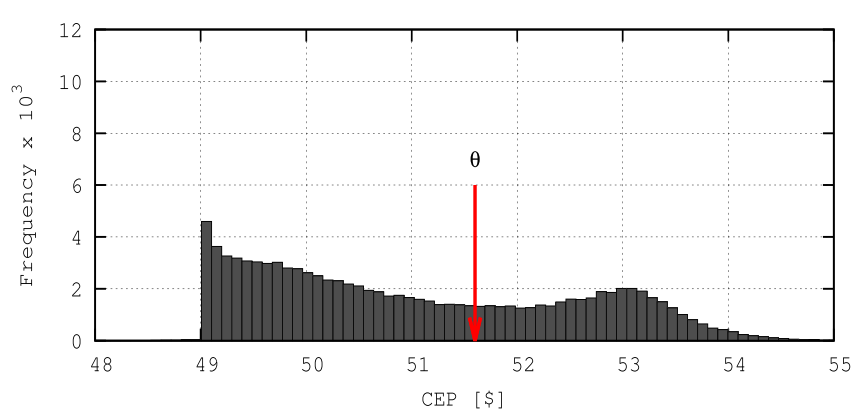

(c) $d_{w}=8 \%$

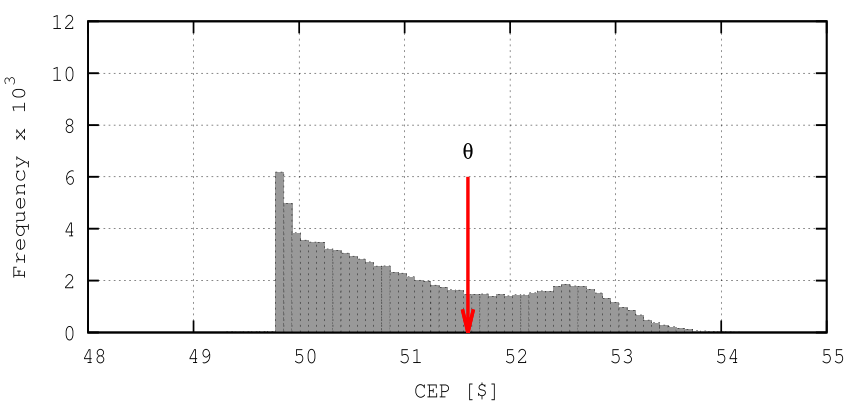

(b) $d_{w}=11 \%$

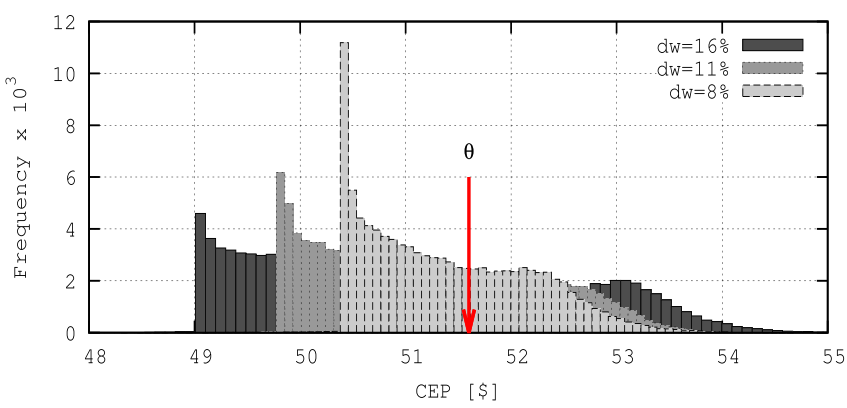

(d) Cost distributions for three different portfolios.

Fig. 9 Comparison of cost of electricity procurement for three different risk profiles: a non risk assessment, $\mathbf{b}$ Excess Cost and $\mathbf{c} \mathrm{CVaR}$ with $\theta=51.6 \$$ per hour and $\alpha=0.7$. Graph $\mathbf{d}$, shows the relative spread among the three risk profiles

can be seen by contrasting the three portfolios: no risk assessment, Excess Cost and CVaR, i.e., $d w=16 \%, 11 \%$ and $8 \%$, respectively.

\section{Conclusion}

This work presented a theoretical approach to electricity procuring using a Ornstein-Uhlenbeck and Lévy process. It studies the energy procurement problem with renewable energy sources. The methodology presented is readily implementable and customizable to serve an LSE in defining contracting strategies in deregulated markets.

With respect to the research in this field and the methods therein this work advances the state-of-the-art by introducing a differential model underlaid by stochastic processes rather than relying on past or given scenarios.

The formulation addresses the merge of renewable and conventional sources with real-time trading. The SPM is composed of 1) A mean-reverting Lévy process and; 2) A Brownian process with multiplicative noise. The former models the sudden changes of electricity spot prices. The latter is used to model the output of a WPP by transforming the drift and diffusion terms to follow a RD. The SPM is straightforwardly scalable and can incorporate other energy sources.
The SPM was complemented with two risk metrics: CVaR and Excess Cost. The paper formulated Excess Cost risk metric as a user defined risk threshold. In the simulations, the threshold is proportional to the dispatchable source. The algorithm outputs the feasible portfolios to hedge against market's volatility and weather uncertainty. The results are general in the sense that they provide an answer irrespective of the policy towards risk.

To validate the SPM we used numerical simulations. A highly liquid market was used to reference the system price for conventional generation, i.e., 24\$. The results show that a LSE should contract between $8 \%$ and $16 \%$ wind power from a WPP with an autocorrelation decay rate $\alpha=0.7$ and nameplate capacity of $1.3 \mathrm{MW}$. The allocated wind share will follow the LSE risk policy. Thus the lower bound guarantees on average the portfolio with the minimum con- ditional-value-at-risk. The risk analysis quantitatively provides decision-making intelligence on the statistical moments of costlier portfolios as a result of the intermittency of wind power.

Furthermore, the SPM convergence to an optimal portfolio irrespectively of the WPP characteristic autocorrelation decay rate. In our testbed, on average, the simulation shows a span from $15 \%$ to $18 \%$ wind power.

Future work is aimed at extending the SPM to incorporate the seller's decision process mechanism. With the 
inclusion of the sellers perspective the networking outcomes, of the two-way buyer-seller relation, and its consequences on portfolio creation are important areas for future research.

Acknowledgements This work was partially funded by 1) Waterford Institute of Technology and 2) Science Foundation Ireland via the CONNECT research centre (grant no. 13/RC/2077).

\section{References}

1. Pazheri F, Othman M, Malik N (2014) A review on global renewable electricity scenario. Renew Sustain Energy Rev 31:835-845

2. Borenstein S (2002) The trouble with electricity markets: Understanding California's restructuring disaster. J Econ Perspect 16:191-211

3. Ghiasi M, Jam MI, Teimourian M, Zarrabi H, Yousefi N (2017) A new prediction model of electricity load based on hybrid forecast engine. Int J Ambient Energy 0(0):1-8

4. Lund PD, Lindgren J, Mikkola J, Salpakari J (2015) Review of energy system flexibility measures to enable high levels of variable renewable electricity. Renew Sustain Energy Rev 45:785-807

5. Markowitz H (1952) Portfolio selection. J Financ 7(1):77-91

6. Olaofe ZO, Folly KA (2013) Wind energy analysis based on turbine and developed site power curves: A case-study of Darling City. Renew Energy 53:306-318

7. Pishgar-Komleh S, Keyhani A, Sefeedpari P (2015) Wind speed and power density analysis based on Weibull and Rayleigh distributions (a case study: Firouzkooh county of Iran). Renew Sustain Energy Rev 42:313-322

8. Carta J, Ramírez P, Velázquez S (2009) A review of wind speed probability distributions used in wind energy analysis: Case studies in the Canary Islands. Ren Sus Energy Rev 13:933955

9. Bjorgan R, Song H, Liu CC, Dahlgren R (2000) Pricing flexible electricity contracts. IEEE Trans Power Syst 15:477-482

10. Bushnell JB, Mansur ET, Saravia C (2008) Vertical arrangements, market structure, and competition: An analysis of restructured US electricity markets. Ame Eco Rev 98:237-266

11. Scharff R, Amelin M (2011) A study of electricity market design for systems with high wind power penetration, In: 2011 8th Int Conf on the European, Energy Market (EEM), pp 614-612. IEEE

12. Ghiasi M, Ahmadinia E, Lariche M, Zarrabi H, Simoes R (2018) A new spinning reserve requirement prediction with hybrid model. Smart Sci 6(3):212-221

13. Akbary P, Ghiasi M, Pourkheranjani MRR, Alipour H, Ghadimi N (2017) Extracting appropriate nodal marginal prices for all types of committed reserve, Computational Economics 1-26. https://doi.org/10.1007/s10614-017-9716-2

14. Conejo AJ, Fernandez-Gonzalez JJ, Alguacil N (2005) Energy procurement for large consumers in electricity markets. IEE Proc - Gen Tran and Distribution 152:357-364

15. Chen M (2011)

16. Kneževic G, Nikolovski S, Marić P (2011) Electricity spot market simulation involving bilateral contracts hedging. In: 2011 8th EEM, pp 122-127
17. Jimenez MBJ, Nerves AC (2013) Optimal allocation of spot market and bilateral contract transactions through quantity declaration adjustments, In: 2013 IEEE PES APPEEC, pp 1-6

18. Palamarchuk S (2009) Bilateral contract correction and cancellation in the competitive electricity markets. In: PowerTech IEEE Bucharest, pp 1-5. IEEE

19. Mateus JC, Cuervo P (J2009) Bilateral negotiation of energy contracts from the buyer perspective. In: 2009 IEEE power energy society general meeting, pp 1-7

20. Wu DJ, Kleindorfer P, Zhang JE (2001) Optimal bidding and contracting strategies in the deregulated electric power market: Part II. In: 34th Int Conf on System Sciences, pp 1-10. IEEE

21. Peng W, Yan-Hong W, Hua-Chun Y, Ren-Mu H (2002) Bayes equilibrium on negotiation means transaction of the power market long-term contract. PowerCon 3:1814-1818

22. Freire L, Street A, Lima DA, Barroso LA (2015) A hybrid MILP and Benders decomposition approach to find the nucleolus quota allocation for a renewable energy portfolio. IEEE Trans Power Syst 30:3265-3275

23. Baeyens E, Bitar EY, Khargonekar PP, Poolla K (2013) Coalitional aggregation of wind power. IEEE Trans Power Syst 28:3774-3784

24. International Renewable Energy Agency, Renewable power generation costs in 2017, 2018

25. Watts S (2013) LMP Overview https://pjmadobeconnectcom/ a16103949/p3c7rb6whya/. [Online; accessed 19-Jul-2016]

26. Longoria G, Jiang D, Davy A, Shi L (2016) Wind energy allocation strategies for long-term contracts in open energy markets. In: IEEE international conference on renewable energy research and applications (ICRERA), pp 783-788, 2016

27. Benth FE, Benth JŠ, Koekebakker S (2008) Stochastic Modelling of Electricity and Related Markets. World Scientific, Singapore

28. Lydia M, Kumar SS, Selvakumar AI, Kumar GEP (2014) A comprehensive review on wind turbine power curve modeling techniques. Ren Sust Energ Rev 30:452-460

29. Glasserman P (2003) Monte carlo methods in financial engineering. Springer-Verlag, New York

30. Gardiner C (2009) Stochastic methods: A Handbook for the Natural and Social Sciences. Springer-Verlag, Heidelberg

31. Shreve SE (2004) Stochastic calculus for finance II continuoustime models. Springer, New York

32. Zhang Y, Wang J, Wang X (2014) Review on probabilistic forecasting of wind power generation. Renew Sustain Energy Rev $32: 255-270$

33. Hau E (2013) Wind turbines fundamentals, technologies, application, economics. Springer-Verlag, Heidelberg

34. Zárate-Miñano R, Milano F Construction of SDE-based wind speed models with exponential autocorrelation ERC UCD, 2015. arXiv: 1511.02345

35. Leon-Garcia A (2008) Probability, statistics, and random processes for electrical engineering. Pearson Edu, Upper Saddle River

36. Gökçek M, Bayülken A, Bekdemir Ş (2007) Investigation of wind characteristics and wind energy potential in Kirklareli. Turkey Renew Energy 32:1739-1752

37. Kasdin N (1995) Runge-Kutta algorithm for the numerical integration of stochastic differential equations. J Guid Control Dyn 18:114-120

38. Artzner P, Delbaen F, Eber J, Heath D (1999) Coherent measures of risk. Math Financ 9:203-208

39. Dehlbæk F (2017) National Report Denmark, tech rep. Danish Energy Regulatory Authority, Denmark 